\section{Thrombin generation in cardiovascular disease and mortality - results from the Gutenberg Health Study}

\author{
Pauline C.S. van Paridon, ${ }^{1,2^{*}}$ Marina Panova-Noeva, ${ }^{2 *}$ Rene van Oerle, ${ }^{1}$ Andreas \\ Schulz, ${ }^{3}$ Iris M. Hermanns, ${ }^{3,4}$ Jürgen H. Prochaska, ${ }^{2,3,5}$ Natalie Arnold, ${ }^{3}$ Harald \\ Binder, ${ }^{6,7}$ Irene Schmidtmann, ${ }^{7}$ Manfred E. Beutel, ${ }^{8}$ Norbert Pfeiffer, ${ }^{9}$ Thomas \\ Münzel, ${ }^{5,10}$ Karl J. Lackner, ${ }^{10,11}$ Hugo ten Cate, ${ }^{1,2}$ Philipp S. Wild ${ }^{2,3,10 \#}$ and Henri \\ M.H. Spronk ${ }^{1 \#}$
}

${ }^{1}$ Laboratory for Clinical Thrombosis and Hemostasis, Department of Internal Medicine, Cardiovascular Research Institute Maastricht (CARIM), Maastricht University Medical Center, Maastricht, the Netherlands; ${ }^{2}$ Center for Thrombosis and Hemostasis (CTH), University Medical Center of the Johannes Gutenberg-University Mainz, Germany; ${ }^{3}$ Preventive Cardiology and Preventive Medicine, Center for Cardiology, University Medical Center of the Johannes Gutenberg-University Mainz, Germany; ${ }^{4}$ University of Applied Sciences, Hochschule Fresenius, Idstein, Germany; ${ }^{5}$ Cardiology I, Center for Cardiology, University Medical Center of the Johannes Gutenberg-University Mainz, Germany; ${ }^{6}$ Institute of Medical Biometry and Statistics, Faculty of Medicine and Medical Center, University of Freiburg, Freiburg, Germany; ${ }^{7}$ Institute of Medical Biostatistics, Epidemiology and Informatics, University Medical Center of the Johannes GutenbergUniversity Mainz, Germany; ${ }^{8}$ Department of Psychosomatic Medicine and Psychotherapy, University Medical Center of the Johannes Gutenberg-University Mainz, Germany; ${ }^{9}$ Department of Ophthalmology, University Medical Center of the Johannes Gutenberg-University Mainz, Germany; ${ }^{10}$ DZHK (German Center for Cardiovascular Research), Partner Site RhineMain, Mainz, Germany and ${ }^{11}$ Institute for Clinical Chemistry and Laboratory Medicine, University Medical Center of the Johannes Gutenberg-University Mainz, Germany

${ }^{*} P C S v P$ and MP-N contributed equally as co-first authors.

"PSW and HMHS contributed equally as co-senior authors.

\section{ABSTRACT}

T hrombin generation may be a potential tool to improve risk stratification for cardiovascular diseases. The aim of this study was to explore the relation between thrombin generation and cardiovascular risk factors, cardiovascular diseases, and total mortality. For this study, 5,000 subjects from the population-based Gutenberg Health Study were analyzed in a highly standardized setting. Thrombin generation was assessed by the Calibrated Automated Thrombogram method at 1 and $5 \mathrm{pM}$ tissue factor triggers in platelet-poor plasma. Lag time, endogenous thrombin potential, and peak height were derived from the thrombin generation curve. Sex-specific multivariable linear regression analysis adjusted for age, cardiovascular risk factors, cardiovascular diseases and therapy, was used to assess clinical determinants of thrombin generation. Cox regression models adjusted for age, sex, cardiovascular risk factors and vitamin $\mathrm{K}$ antagonists investigated the association between thrombin generation parameters and total mortality. Lag time was positively associated with obesity and dyslipidaemia for both sexes $(P<0.0001)$. Obesity was also positively associated with endogenous thrombin potential in both sexes $(P<0.0001)$ and peak height in males $(1 \mathrm{pM}$ tissue factor, $P=0.0048)$ and females $(P<0.0001)$. Cox regression models showed an increased mortality in individuals with lag time $(1 \mathrm{pM}$ tissue factor, hazard ratio=1.46, 95\% confidence interval: 1.07-2.00; $P=0.018)$ and endogenous thrombin potential ( $5 \mathrm{pM}$ tissue factor, hazard ratio=1.50, $95 \%$ confidence interval: $1.06-2.13 ; P=0.023$ ) above the $95^{\text {th }}$ percentile of the reference group, independently of the cardiovascular risk profile.
Haematologica 2020

Volume 105(9):2327-2334

\section{Correspondence:}

HENRI M.H. SPRONK

henri.spronk@maastrichtuniversity.n

Received: March 13, 2019.

Accepted: December 3, 2019.

Pre-published: December 5, 2019.

doi:10.3324/haematol.2019.221655

(C)2020 Ferrata Storti Foundation

Material published in Haematologica is covered by copyright. All rights are reserved to the Ferrata Storti Foundation. Use of published material is allowed under the following terms and conditions:

https://creativecommons.org/licenses/by-nc/4.0/legalcode. Copies of published material are allowed for personal or internal use. Sharing published material for non-commercial purposes is subject to the following conditions: https://creativecommons.org/licenses/by-nc/4.0/legalcode, sect. 3. Reproducing and sharing published material for commercial purposes is not allowed without permission in writing from the publisher. 
This large-scale study demonstrates that traditional cardiovascular risk factors, particularly obesity, are relevant determinants of thrombin generation. Lag time and endogenous thrombin potential were found to be potentially relevant predictors of increased total mortality, observations which deserve further investigation.

\section{Introduction}

Thrombin generation (TG) is one of the key enzymatic processes that direct the activity of the hemostatic system and coagulation cascade up to and including the formation of a fibrin clot. ${ }^{1}$ Physiologically, thrombin formation is essential to maintain hemostasis and bleeding tendencies are associated with reduced thrombin (and hence fibrin) formation. ${ }^{2}$ An enhanced plasma potential to generate thrombin has been linked to an increased risk of venous thromboembolism, while the associations with arterial vascular disease are still inconsistent. ${ }^{3.8}$ The TG assay is an important method addressing the overall potential of a plasma sample to form thrombin. More than $95 \%$ of TG occurs after initial formation of fibrin, so routine diagnostic coagulation tests, such as prothrombin time and activated partial thromboplastin time fail to reproduce this overall potential. Hence, there is a strong research interest in TG as a promising diagnostic tool for hypo- and particularly hyper-coagulability phenotypes. ${ }^{9}$

In a study of healthy individuals, fibrinogen, factor XII, free tissue factor pathway inhibitor (TFPI) and antithrombin have been identified as major determinants of TG parameters. ${ }^{10}$ Relation to demographic characteristics, such as age and sex, has been previously addressed but the studies have been small and results are not entirely consistent. ${ }^{11,12}$ As TG analysis is a promising tool to estimate a subject's risk for thrombosis or more broadly cardiovascular diseases (CVD), it is of eminent importance to fully understand the nature and direction of effects of cardiovascular risk factors (CVRF).

Hence, we undertook the present investigation in the first 5,000 participants of the population-based Gutenberg Health Study. The primary aim of this study was to investigate CVRF and CVD as major clinical determinants of increased TG in a large population-based sample. Additional aims were to obtain age- and sex-related reference values for TG parameters in a representative subsample of adults who were healthy from a cardiovascular point of view. Finally, having prospective data on total mortality allowed us to investigate the relation between TG parameters and all-cause mortality.

\section{Methods}

\section{Research design}

The Gutenberg Health Study, a population-based, prospective, observational, single-center cohort study in the Rhine-Main region in Western Mid-Germany, was designed to improve the individual risk prediction of CVD. At baseline examination, the study included a total of 15,010 individuals. A detailed description of the research design is provided in Online Supplementary Material, Part A. Further details of the study protocol and purpose are discussed elsewhere. ${ }^{13}$

The study was designed in accordance with the tenets of the revised Helsinki protocol, and the protocol and sampling design were approved by the local ethics committee. The sampling design was additionally approved by local and state data safety commissioners.

\section{Study sample and reference sample}

The study sample consisted of the first 5,000 subjects enrolled into the Gutenberg Health Study between April 2007 and October 2008. After excluding subjects without biomaterial available or without complete TG assessment (one or several TG parameters missing), 4,843 individuals were successfully included in the overall study sample for the present analysis.

The reference group was defined as subjects apparently healthy from a cardiovascular point of view, without a history of CVD (myocardial infarction, congestive heart failure, coronary artery disease, venous thromboembolism, atrial fibrillation or peripheral artery disease), presence of CVRF (obesity, dyslipidemia, arterial hypertension, diabetes mellitus) or use of antithrombotic agents, oral contraceptives or hormonal replacement therapy. In addition, individuals with a self-reported history of inherited coagulation abnormalities were excluded from the reference sample. A detailed definition of traditional CVRF and categorization of medications are provided in Online Supplementary Material, Part A.

\section{Clinical assessment and laboratory measurements}

Clinical examination and determination of CVRF were performed as published elsewhere..$^{14,15}$ Standard laboratory measurements were carried out at the Institute of Clinical Chemistry and Laboratory Medicine, University Medical Center Mainz, Germany. Details on venous blood sampling and plasma preparation are provided in Online Supplementary Material, Part A. TG was assessed according to the recommendations ${ }^{16}$ for the Calibrated Automated Thrombogram (CAT) assay (Thrombinoscope BV, Maastricht, the Netherlands) in plateletpoor plasma. The TG was triggered by $1 \mathrm{pM}$ tissue factor (TF) with $4 \mu \mathrm{M}$ phospholipids at 20:20:60 mol\% phosphatidylserine/phosphatidylethanolamine/phosphatidylcholine, or $5 \mathrm{pM}$ TF with $4 \mu \mathrm{M}$ phospholipids. Trigger reagents were selected for commercial availability, e.g. PPP Reagent and PPP Low Reagent. The CAT method employs a low affinity fluorogenic substrate for thrombin (Z-Gly-Gly-Arg-AMC) in order to monitor thrombin activity continuously in clotting plasma. TG measurements were calibrated against the fluorescence curve obtained in a sample from the same plasma $(80 \mu \mathrm{L})$, supplemented with a fixed amount of thrombin- $\alpha 2$-macroglobulin complex $(20 \mu \mathrm{L}$ of Thrombin Calibrator; Thrombinoscope BV, Maastricht, the Netherlands) and $20 \mu \mathrm{L}$ of the fluorogenic substrate and calcium chloride mixture. ${ }^{16}$ TG parameters were derived from the TG curve and include lag time (time to minimum thrombin formed, in min), peak height (the maximum amount of thrombin formed, in $\mathrm{nM}$ ) and endogenous thrombin potential (ETP or area under the curve, in nM.min). All samples were tested as one batch using one batch of reagents within a period of 6 months. Two technicians performed the analyses on three validated systems and normal pooled plasma was included in each assay run for in-house quality control according to our ISO9001 certification (Coagulation Profile BV, Maastricht, the Netherlands). 
Data management and statistical analysis are described in Online Supplementary Material, Part A.

\section{Results}

Study sample and reference subsample characteristics

While there was a balanced sex ratio in the overall study sample, there was a slight preponderance of women $(55.3 \%)$ in the reference subsample. The median and interquartile range (IOR) of age of the study sample was 56 years (IOR, 46-66) in males and 55 years (IOR, 45-64) in females. The reference sample included 1,210 subjects, of whom 541 (44.7\%) were male and 669 (55.3\%) female. The median age of the reference sample was 47 years (IOR, 42-55) in males and 48 years (IOR, 41-54) in females. In the study population, hypertension was the most prevalent CVRF, being present in $56.6 \%$ of the male population and $46.1 \%$ of the female population, followed by dyslipidemia. Antithrombotic agents were taken by $15.9 \%$ of males and $9.4 \%$ of females. Among females, $6.4 \%$ were taking oral contraceptives and $12.3 \%$ hormone replacement therapy. Detailed characteristics of the study population and reference sample are presented in Online Supplementary Table S1.

\section{Thrombin generation reference values and parameters in the overall study sample}

The results of the TG parameters in males and females from the study sample and reference subsample (reference values) are shown in Table 1 . Females presented with a shorter lag time at $1 \mathrm{pM}$ TF and $5 \mathrm{pM}$ TF $(P<0.0001$ for both), higher ETP at 1 pM TF $(P<0.0001)$ and higher peak height at 1 pM TF $(P=0.014)$ and at 5 pM TF $(P<0.0001)$ compared to males from the reference subsample. In the study sample, females presented with a shorter lag time at 1 pM TF $(P<0.0001)$, as well as higher ETP and peak height at both 1 pM TF and 5 pM TF $(P<0.0001$ for both), compared to males.

\section{Clinical determinants of thrombin generation in the overall study sample}

As shown in Table 2, age was associated with longer lag time, both in males (Table $2 \mathrm{~A}$ ) and females (Table 2B), at 1 pM TF (males: $P=0.014$; females: $P<0.0001$ ) and at 5 pM TF (Online Supplementary Table S2A, B). In males, age was pos- itively associated with ETP at $1 \mathrm{pM} \mathrm{TF}$ (Table 2A) and peak height at $1 \mathrm{pM}$ TF (Table 2A) and at $5 \mathrm{pM}$ TF (Online Supplementary Table S2A). In contrast, in females, age was associated with lower ETP at $1 \mathrm{pM} \mathrm{TF}(P=0.015)$ and lower peak height at $5 \mathrm{pM}$ TF (Online Supplementary Table S2B).

Of the various CVRF considered, obesity showed a positive association with lag time (males: $P<0.0001$; females: $P<0.0001)$, ETP (males: $P<0.0001$; females: $P<0.0001$ ) and peak height (males: $P=0.0048$; females: $P<0.0001$ ) at $1 \mathrm{pM}$ TF. Dyslipidemia was positively associated with lag time in both males $(P<0.0001)$ and females $(P<0.0001)$ and with ETP in males only $(P=0.0057)$ at 1 pM TF. Similar findings for both obesity and dyslipidemia were observed at $5 \mathrm{pM}$ TF as shown in Online Supplementary Table S2A, B. No associations were found for TG and history of CVD.

\section{Therapeutic agents and thrombin generation parameters in the overall study sample}

Females using oral contraceptives or hormone replacement therapy presented with shorter lag time, higher ETP and peak height at both $1 \mathrm{pM} \mathrm{TF}$ (Table 2B) and 5 pM TF (Online Supplementary Table S2B).

Use of vitamin $\mathrm{K}$ antagonists reduced TG as shown by prolonged lag time, and reduced ETP and peak height (Table $2 \mathrm{~A}, \mathrm{~B})$. These effects were detectable at $1 \mathrm{pM}$ TF in both males $(P<0.0001$; ETP: $P<0.0001$; peak height: $P<0.0001)$ and females $(P<0.0001$; ETP: $P<0.0001$; peak height: $P<0.0001$;). Similar results were obtained at $5 \mathrm{pM}$ TF (Online Supplementary Table S2A, B).

Results from multiple linear regression analysis for $\mathrm{TG}$ parameters at $1 \mathrm{pM}$ TF and at $5 \mathrm{pM}$ TF, demonstrated associations between other medications, in addition to vitamin $\mathrm{K}$ antagonists, and TG as shown in Table 3A, B. The lag time at 1 pM TF was positively associated with intake of cardiac drugs $(P<0.0001)$, diuretics $(P=0.00043)$, anti-gout preparations $(P=0.00038)$ and immunosuppressants $(P=0.00021)$, and inversely associated with hormone-containing drugs (i.e., hormone replacement therapy and oral contraceptives, $P<0.0001)$. Differently, ETP was inversely associated with cardiac drugs, ATC code C01 $(P<0.0001)$ at 5 pM TF and positively with hormonecontaining drugs $(P<0.0001)$. Peak height showed a positive association with hormone-containing drugs $(P<0.0001)$. The results at $5 \mathrm{pM}$ TF were comparable to the results at $1 \mathrm{pM}$ TF.

Table 1. Parameters of thrombin generation in the reference subsample and the study sample.

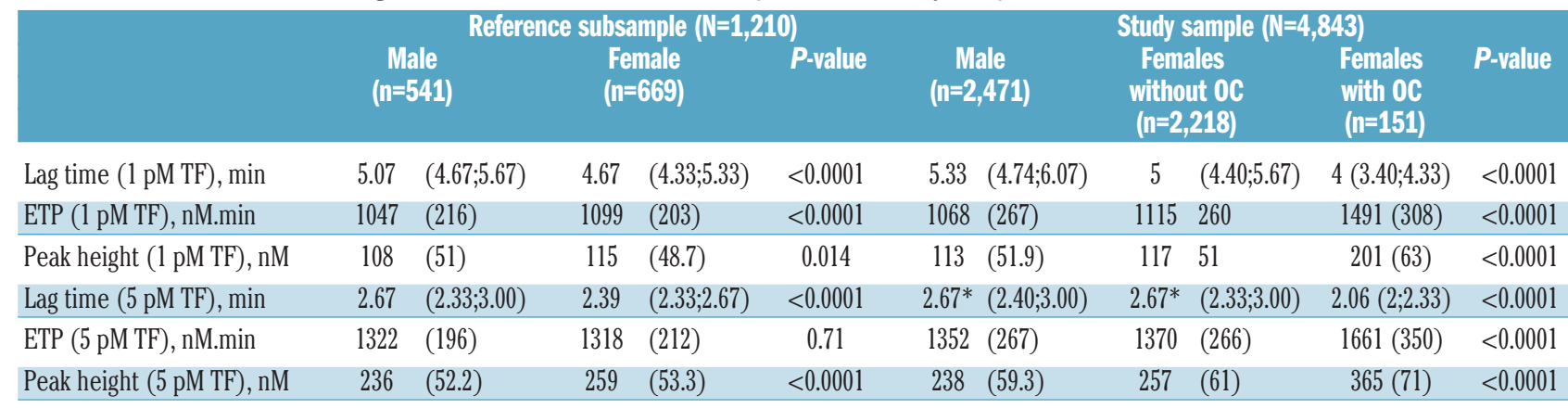

The values presented are thrombin generation parameters at $1 \mathrm{pM}$ and $5 \mathrm{pM}$ tissue factor in the reference subsample and study sample. Medians (interquartile range) of lag time and means (standard deviation) of endogenous thrombin potential and peak height are presented. *Due to equal times, the median values in males and females are the same; however, the distribution of the lag time values is different in males and females.TF: tissue factor; ETP: endogenous thrombin potential. 
Kaplan-Meier survival curves and Cox-regression models

During the follow-up period until April 2017, with a median follow-up of 9.21 (8.83-9.65) years, a total of 308 deaths were registered. As presented in Figure 1, a longer lag time above the $90^{\text {th }}$ percentile of the reference, at both $1 \mathrm{pM} \mathrm{TF}$ (Figure 1A) and 5 pM TF (Figure 1B), was signif- icantly associated with worse survival $(P<0.0001)$. In addition, higher ETP above the $90^{\text {th }}$ percentile $(P=0.034)$ and 97. $5^{\text {th }}$ percentile $(P=0.00097)$ of the reference sample (Figure 1C), measured at $5 \mathrm{pM}$ TF was associated with worse survival. No such associations were observed for ETP at 1 pM TF.

Table 2A. Multivariable linear regression in the overall study sample for parameters of thrombin generation in males at $1 \mathrm{pM}$ tissue factor.

\begin{tabular}{|c|c|c|c|c|c|c|c|c|c|c|}
\hline \multirow[t]{2}{*}{ Variable } & \multirow[t]{2}{*}{ Males (N) } & \multicolumn{3}{|c|}{ Log (lag time [min]) } & \multicolumn{3}{|c|}{ EIP (nM.min) } & \multicolumn{3}{|c|}{ Peak height (nM) } \\
\hline & & $\beta$ & $95 \%$ Cl & P-value & $\beta$ & $95 \%$ CI & P-value & $\beta$ & $95 \% \mathrm{Cl}$ & P-value \\
\hline Age (10 years) & & 0.0098 & $(0.00199 ; 0.0175)$ & 0.014 & 28.9 & $(17.9 ; 39.9)$ & $<0.0001$ & 7.60 & $(5.23 ; 9.97)$ & $<0.0001$ \\
\hline Diabetes & 242 & -0.0318 & $(-0.0593 ;-0.00441)$ & 0.023 & -6.00 & $(-45.0 ; 33.0)$ & 0.76 & 5.16 & $(-3.21 ; 13.5)$ & 0.23 \\
\hline Obesity & 628 & 0.0392 & $(0.0214 ; 0.0570)$ & $<0.0001$ & 54.7 & $(29.5 ; 80.0)$ & $<0.0001$ & 7.83 & $(2.40 ; 13.3)$ & 0.0048 \\
\hline Smoking & 515 & 0.0151 & $(-0.00328 ; 0.0334)$ & 0.11 & 18.2 & $(-7.82 ; 44.3)$ & 0.17 & -1.64 & $(7.24 ; 3.96)$ & 0.57 \\
\hline Hypertension & 1,395 & 0.0135 & $(-0.00263 ; 0.0297)$ & 0.10 & -6.70 & $(29.7 ; 16.3)$ & 0.57 & -3.22 & $(-8.15 ; 1.72)$ & 0.20 \\
\hline Dyslipidemia & 917 & 0.0428 & $(0.0275 ; 0.0580)$ & $<0.0001$ & 30.6 & $(8.96 ; 52.3)$ & 0.0057 & 1.39 & $(-3.27 ; 6.04)$ & 0.56 \\
\hline FH of MI/stroke & 853 & -0.00624 & $(-0.0217 ; 0.00919)$ & 0.43 & 17.1 & $(-4.84 ; 39.0)$ & 0.13 & -0.283 & $(-4.99 ; 4.42)$ & 0.91 \\
\hline History of MI & 116 & 0.0263 & $(-0.0193 ; 0.0719)$ & 0.26 & -57.5 & $(-122 ; 7.32)$ & 0.082 & -8.36 & $(-22.3 ; 5.56)$ & 0.24 \\
\hline History of stroke & 57 & 0.0352 & $(-0.0169 ; 0.0874)$ & 0.19 & -39.7 & $(-114 ; 34.4)$ & 0.29 & -2.67 & $(-18.6 ; 13.2)$ & 0.74 \\
\hline History of CAD & 167 & -0.0306 & $(-0.0674 ; 0.00617)$ & 0.10 & 25.7 & $(-26.6 ; 77.9)$ & 0.34 & 1.21 & $(-10.0 ; 12.4)$ & 0.83 \\
\hline History of AF & 93 & 0.0418 & $(-0.00183 ; 0.0854)$ & 0.061 & -19.5 & $(-81.4 ; 42.4)$ & 0.54 & -6.31 & $(-19.6 ; 6.99)$ & 0.35 \\
\hline History of PAD & 107 & 0.0367 & $(-0.00267 ; 0.0761)$ & 0.068 & -53.0 & $(-109 ; 2.95)$ & 0.064 & -10.6 & $(-22.6 ; 1.40)$ & 0.083 \\
\hline History of VTE & 71 & -0.0342 & $(-0.0794 ; 0.0111)$ & 0.14 & -4.91 & $(-69.2 ; 59.4)$ & 0.88 & -1.44 & $(-15.3 ; 12.4)$ & 0.84 \\
\hline History of CHF & 39 & -0.00240 & $(-0.0652 ; 0.0604)$ & 0.94 & -15.1 & $(-104 ; 74.0)$ & 0.74 & 5.46 & $(-13.7 ; 24.6)$ & 0.58 \\
\hline History of cancer & 194 & -0.000508 & $(-0.0284 ; 0.0273)$ & 0.11 & 0.764 & $(-38.8 ; 40.3)$ & 0.97 & -2.11 & $(-10.6 ; 6.39)$ & 0.63 \\
\hline VKA use & 391 & 0.646 & $(0.0589 ; 0.702)$ & $<0.0001$ & -716 & $(-796 ;-637)$ & $<0.0001$ & -66.3 & $(-83.5 ;-49.2)$ & $<0.0001$ \\
\hline
\end{tabular}

Multivariable linear regression models were calculated in the overall study sample for each parameter of thrombin generation as a dependent variable separately.The analysis was adjusted for age, vitamin $\mathrm{K}$ antagonist use,presence of cardiovascular risk factors and cardiovascular diseases. ETP: endogenous thrombin potential; $95 \%$ CI:95\% confidence interval; FH: family history; MI: myocardial infarction; CAD: coronary artery disease; AF: atrial fibrillation; PAD: peripheral artery disease;VTE: venous thromboembolism; CHF: congestive heart failure; $\mathrm{VKA}$ : vitamin $\mathrm{K}$ antagonist.

Table 2B. Multivariable linear regression in the overall study sample for parameters of thrombin generation in females at $1 \mathrm{pM}$ tissue factor.

\begin{tabular}{|c|c|c|c|c|c|c|c|c|c|c|}
\hline Variable & imales (N) & & Log (lag time [min & & & $\operatorname{ETP}(\mathrm{nM} \cdot \mathrm{min})$ & & & sak height (n) & \\
\hline & & $\beta$ & $95 \%$ Cl & P-value & $\beta$ & $95 \%$ Cl & P-value & $\beta$ & $95 \%$ Cl & P-value \\
\hline Age (10 years) & & 0.0410 & $(0.0332 ; 0.0488)$ & $<0.0001$ & -15.5 & $(-28.0 ;-2.99)$ & 0.015 & 0.657 & $(-1.90 ; 3.22)$ & 0.62 \\
\hline Diabetes & 123 & -0.00149 & $(-0.0342 ; 0.0312)$ & 0.93 & -34.2 & $(86.8 ; 18.3)$ & 0.20 & 2.24 & $(-8.54 ; 13.0)$ & 0.68 \\
\hline Obesity & 542 & 0.0587 & $(0.0411 ; 0.0763)$ & $<0.0001$ & 110 & $(81.9 ; 139)$ & $<0.0001$ & 11.7 & $(5.86 ; 17.5)$ & $<0.0001$ \\
\hline Smoking & 415 & 0.0222 & $(0.00372 ; 0.0408)$ & 0.019 & -17.3 & $(-47.1 ; 12.4)$ & 0.25 & -7.23 & $(-13.3 ;-1.12)$ & 0.020 \\
\hline Hypertension & 1,092 & 0.0214 & $(0.00582 ; 0.0370)$ & 0.0072 & -14.7 & $(-39.8 ; 10.4)$ & 0.25 & -4.76 & $(-9.90 ; 0.390)$ & 0.070 \\
\hline Dyslipidemia & 517 & 0.0429 & $(0.0254 ; 0.0604)$ & $<0.0001$ & 24.5 & $(-3.63 ; 52.6)$ & 0.088 & -2.02 & $(-7.80 ; 3.75)$ & 0.49 \\
\hline FH of MI/stroke & 924 & -0.0089 & $(-0.0233 ; 0.00546)$ & 0.22 & 5.08 & $(-18.0 ; 28.1)$ & 0.67 & 1.50 & $(-3.24 ; 6.23)$ & 0.54 \\
\hline History of MI & 35 & -0.0273 & $(-0.099 ; 0.0445)$ & 0.46 & -19.2 & $(-135 ; 96.2)$ & 0.74 & 8.04 & $(-15.6 ; 31.7)$ & 0.51 \\
\hline History of stroke & 35 & -0.0826 & $(-0.149 ;-0.0159)$ & 0.015 & 105 & $(-2.54 ; 212)$ & 0.056 & 21.2 & $(-0.799 ; 43.2)$ & 0.059 \\
\hline History of CAD & 50 & 0.0336 & $(-0.0178 ; 0.0850)$ & 0.20 & -46.4 & $(-129 ; 36.1)$ & 0.27 & 3.34 & $(-13.6 ; 20.3)$ & 0.70 \\
\hline History of AF & 35 & -0.0320 & $(-0.106 ; 0.0417)$ & 0.39 & 104 & $(-14.8 ; 222)$ & 0.086 & 20.3 & $(-3.95 ; 44.6)$ & 0.10 \\
\hline History of PAD & 93 & 0.0390 & $(0.00303 ; 0.0749)$ & 0.034 & -25.1 & $(-82.9 ; 32.6)$ & 0.39 & -3.96 & $(-15.8 ; 7.89)$ & 0.51 \\
\hline History of VTE & 124 & -0.0348 & $(-0.0687 ;-0.000971)$ & 0.044 & 44.4 & $(-10.0 ; 98.8)$ & 0.11 & 1.70 & $(-9.47 ; 12.9)$ & 0.77 \\
\hline History of CHF & 36 & -0.0588 & $(-0.13 ; 0.0122)$ & 0.10 & -3.77 & $(-118 ; 110)$ & 0.95 & -9.50 & $(32.9 ; 13.9)$ & 0.43 \\
\hline History of cancer & 236 & 0.00886 & $(-0.015 ; 0.0327)$ & 0.47 & -33.6 & $(-71.9 ; 4.75)$ & 0.086 & -3.68 & $(-11.5 ; 4.19)$ & 0.36 \\
\hline HRT & 223 & -0.0457 & $(-0.0669 ;-0.0246)$ & $<0.0001$ & 60.1 & $(26.2 ; 94.1)$ & 0.00053 & 14.3 & $(7.28 ; 21.2)$ & $<0.0001$ \\
\hline Oral contraceptives & 151 & -0.145 & $(-0.175 ;-0.116)$ & $<0.0001$ & 379 & $(331 ; 427)$ & $<0.0001$ & 91.1 & $(81.3 ; 101)$ & $<0.0001$ \\
\hline VKA use & 292 & 0.816 & $(0.743 ; 0.889)$ & $<0.0001$ & -751 & $(-869 ;-633)$ & $<0.0001$ & -92.1 & $(-116 ;-67.9)$ & $<0.0001$ \\
\hline
\end{tabular}

Multivariable linear regression models were calculated in the overall study sample for each parameter of thrombin generation as a dependent variable separately. The analysis was adjusted for age, use of vitamin $\mathrm{K}$ antagonists, oral contraceptives, and hormone replacement therapy, cardiovascular risk factors and cardiovascular diseases. ETP: endogenous thrombin potential; $95 \% \mathrm{CI}$ : 95\% confidence interval; FH: family history; MI: myocardial infarction; CAD: coronary artery disease; $\mathrm{AF}$ : atrial fibrillation; PAD: peripheral artery disease;VTE: venous thromboembolism; CHF: congestive heart failure; HRT: hormone replacement therapy;VKA: vitamin K antagonist. 
Considering the strong positive association between TG and the use of oral contraceptives or hormone replacement therapy, individuals taking these medications were excluded from Cox regression analysis. In the first model, adjustments were made for age, sex, and vitamin $\mathrm{K}$ antagonist use, whereas in the second and third models, additional adjustments were made for CVRF and CVD, respectively. As demonstrated by the second model in Table 4A, $B$, Cox regression analysis confirmed an increased mortality for individuals with a lag time at $1 \mathrm{pM}$ TF above the $95^{\text {th }}$ percentile of the reference [hazard ratio $(\mathrm{HR})=1.55$, 95\% confidence interval (95\% CI): 1.14-2.11; $P=0.0058]$ and with ETP at $5 \mathrm{pM} \mathrm{TF}$ above the $95^{\text {th }}$ percentile of the reference ( $\mathrm{HR}=1.53$, 95\% CI: 1.09-2.15; $P=0.015)$, independently of the presence of CVRF. After additional adjustment for CVD, lag time at $1 \mathrm{pM}$ TF ( $\mathrm{HR}=1.46$, 95\% CI: $1.07-2.00 ; P=0.018)$ and ETP at $5 \mathrm{pM}$ TF $(\mathrm{HR}=1.50$, 95\% CI: 1.06-2.13; $P=0.023)$ remained associated with mortality.

\section{Discussion}

The formation of thrombin is one of the key processes underlying thrombotic diseases and its role in CVD due to atherosclerosis attracted new interest with recent data showing superior efficacy of a combined strategy of aspirin and low-dose direct oral anticoagulation in reducing atherothrombotic events. ${ }^{17}$ Hence, limiting TG by inhibiting factor Xa provides an interesting approach to lower cardiovascular risk. In this study we explore the clinical determinants of TG measured in plasma, in a large population-derived study. Our data provide important insights into the effects of CVRF in males and females. This study is the first to demonstrate the positive association of TG parameters, ETP as a global measure of procoagulant and anticoagulant action in plasma and lag time, with total mortality, independent of age, sex and CVRF.

The presented reference values may be generalized to other laboratories. However, the reference ranges should be used with caution as the (pre-)analytical conditions of the assay may influence the reference ranges and standardization between laboratories is needed, as well as confirmation of the observed data. The reference values of the TG parameters as well as the mean and median values of the TG parameters in the overall study sample showed sex-specific differences with females having shorter lag times and higher ETP and peak height, compared to males. The sex differences in TG can be partly explained by the strong influence of female endogenous sex hormones on the coagulation cascade, as higher levels of fibrinogen and lower levels of protein $\mathrm{S}$, antithrombin and protein $\mathrm{C}$ were observed in females, compared to males, irrespective of hormonal treatment. ${ }^{18}$

Following a sex-stratified, fully adjusted, large, multivariable model analysis we show that age, obesity and dyslipidemia are the most important clinical factors linked with higher TG potential. Furthermore, this study demonstrates the effect of different groups of medication on TG, with hormone-containing drugs being positively associated and anticoagulant and antiarrhythmic drugs being inversely associated with TG potential.

Few studies have described the effect of age on TG parameters. ${ }^{10,11,19}$ Collectively, these studies suggest that TG potential enhances with increasing age, indicated by shorter lag time and higher ETP and peak height.

Table 3A. Multiple linear regression analysis of the effects of drugs on thrombin generation parameters in the study sample at $1 \mathrm{pM}$ tissue factor.

\begin{tabular}{|c|c|c|c|c|c|c|c|c|c|}
\hline \multirow[b]{2}{*}{ Drug } & \multicolumn{3}{|c|}{ Log (lag time [min]) } & \multicolumn{3}{|c|}{ ETP (nM.min) } & \multicolumn{3}{|c|}{ Peak height (nM) } \\
\hline & $\beta$ & $95 \% \mathrm{Cl}$ & P-value & $\beta$ & $95 \%$ CI & P-value & $\beta$ & $95 \%$ Cl & P-value \\
\hline $\begin{array}{l}\text { Sex hormones } \\
\text { and modulators of the } \\
\text { genital system }\end{array}$ & -0.0795 & $(-0.0979 ;-0.0611)$ & $<0.000001$ & 151 & $(124 ; 178)$ & $<0.000001$ & 35.1 & $(29.7 ; 40.4)$ & $<0.000001$ \\
\hline Antithrombotic agents & 0.0862 & $(0.0661 ; 0.106)$ & $<0.000001$ & -92.8 & $(-122 ;-63.4)$ & $<0.000001$ & -5.01 & $(-11.0 ; 0.952)$ & 0.10 \\
\hline Cardiac therapy & 0.112 & $(0.0763 ; 0.147)$ & $<0.000001$ & -83.3 & $(-135 ;-31.5)$ & 0.0016 & -3.94 & $(-14.4 ; 6.54)$ & 0.46 \\
\hline Immunosuppressants & 0.114 & $(0.0537 ; 0.174)$ & 0.00021 & -28.6 & $(-116 ; 59.0)$ & 0.52 & -1.37 & $(-19.1 ; 16.3)$ & 0.88 \\
\hline Anti-gout preparations & 0.0552 & $(0.0248 ; 0.0856)$ & 0.00038 & 0.270 & $(-44.1 ; 44.6)$ & 0.99 & 3.76 & $(-5.20 ; 12.7)$ & 0.41 \\
\hline
\end{tabular}

The analysis was adjusted for age, sex, cardiovascular risk factors and cardiovascular diseases. Bonferroni corrected $P$-value (0.00079) is used. For categorization of medication groups see Online Supplementary Material Part A. ETP: endogenous thrombin potential; 95\% CI: 95\% confidence interval.

Table 3B. Multiple linear regression analysis of the effects of drugs on thrombin generation parameters in the study sample at $5 \mathrm{pM}$ tissue factor.

\begin{tabular}{|c|c|c|c|c|c|c|c|c|c|}
\hline \multirow[b]{2}{*}{ Drug } & \multicolumn{3}{|c|}{ Log (lag time [min]) } & \multicolumn{3}{|c|}{ EIP (nM.min) } & \multicolumn{3}{|c|}{ Peak height (nM) } \\
\hline & $\beta$ & $95 \%$ Cl & P-value & $\beta$ & $95 \%$ CI & P-value & $\beta$ & $95 \%$ Cl & P-value \\
\hline $\begin{array}{l}\text { Sex hormones and } \\
\text { modulators of the } \\
\text { genital system }\end{array}$ & -0.0558 & $(-0.0706 ;-0.0411)$ & $<0.000001$ & 120 & $(93.6 ; 147)$ & $<0.000001$ & 44.9 & $(38.7 ; 51.1)$ & $<0.000001$ \\
\hline Antithrombotic agents & 0.0656 & $(0.0495 ; 0.0817)$ & $<0.000001$ & -122 & $(-151 ;-93.4)$ & $<0.000001$ & -19.4 & $(-26.3 ;-12.6)$ & $<0.000001$ \\
\hline Cardiac therapy & 0.0916 & $(0.0633 ; 0.120)$ & $<0.000001$ & -122 & $(-173 ;-71.1)$ & 0.0000028 & -16.0 & $(-28.0 ;-3.89)$ & 0.0096 \\
\hline Diuretics & 0.0354 & $(0.0157 ; 0.0522)$ & 0.00043 & -37.0 & $(-72.5 ;-1.45)$ & 0.041 & -4.55 & $(-12.9 ; 3.84)$ & 0.29 \\
\hline
\end{tabular}

The analysis was adjusted for age, sex, cardiovascular risk factors and cardiovascular diseases. Bonferroni corrected $P$-value $(0.00079)$ is used. For categorization of medication groups see Online Supplementary Material Part A. ETP: endogenous thrombin potential; 95\% CI: 95\% confidence interval. 
Table 4A. Prognostic value of markers of thrombin generation, measured at $1 \mathrm{pM}$ tissue factor, for mortality.

\begin{tabular}{|c|c|c|c|c|c|c|c|c|c|}
\hline \multirow[t]{2}{*}{ TG parameter } & \multicolumn{3}{|c|}{ First mode** } & \multicolumn{3}{|c|}{ Second model** } & \multicolumn{3}{|c|}{ Third model*** } \\
\hline & HR & $95 \%$ CI & P-value & HR & $95 \% \mathrm{Cl}$ & P-value & HR & $95 \%$ CI & P-value \\
\hline Lag time below $5 \%$ of reference & 1.22 & $(0.58 ; 2.60)$ & 0.60 & 1.11 & $(0.52 ; 2.37)$ & 0.78 & 1.14 & $(0.53 ; 2.42)$ & 0.74 \\
\hline Lag time above $95 \%$ of reference & 1.54 & $(1.14 ; 2.08)$ & 0.0055 & 1.55 & $(1.14 ; 2.11)$ & 0.0058 & 1.46 & $(1.07 ; 2.00)$ & 0.018 \\
\hline ETP below $5 \%$ of reference & 1.08 & $(0.75 ; 1.56)$ & 0.66 & 1.11 & $(0.77 ; 1.60)$ & 0.58 & 1.05 & $(0.72 ; 1.52)$ & 0.80 \\
\hline ETP above $95 \%$ of reference & 1.41 & $(0.98 ; 2.03)$ & 0.061 & 1.44 & $(0.99 ; 2.07)$ & 0.051 & 1.41 & $(0.97 ; 2.04)$ & 0.071 \\
\hline Peak height below $5 \%$ of reference & 1.14 & $(0.78 ; 1.67)$ & 0.51 & 1.18 & $(0.81 ; 1.74)$ & 0.39 & 1.12 & $(0.76 ; 1.65)$ & 0.56 \\
\hline Peak height above $95 \%$ of reference & 1.28 & $(0.82 ; 2.01)$ & 0.27 & 1.29 & $(0.83 ; 2.03)$ & 0.26 & 1.39 & $(0.89 ; 2.18)$ & 0.15 \\
\hline
\end{tabular}

Cox regression model performed in the overall study sample with mortality as outcome and markers of thrombin generation as predictors. Models were *adjusted for age, sex and vitamin K antagonist (VKA) use. **adjusted for age, sex,VKA use and presence of cardiovascular risk factors (CVRF). ***adjusted for age, sex,VKA, use, presence of CVRF, and cardiovascular disease.TG: thrombin generation; HR: hazard ratio; $95 \%$ CI: 95\% confidence interval; ETP: endogenous thrombin potential.

Table 4B. Prognostic value of markers of thrombin generation, measured at $5 \mathrm{pM}$ tissue factor, for mortality.

\begin{tabular}{lccccccccc}
\hline TG parameter & HR & $\begin{array}{c}\text { First mode** } \\
95 \% \text { CI }\end{array}$ & P-value & HR & $\begin{array}{c}\text { Second model** } \\
95 \% \text { CI }\end{array}$ & P-value & HR & $\begin{array}{c}\text { Third model*** } \\
95 \% \text { CI }\end{array}$ & P-value \\
Lag time below 5\% of reference & 1.08 & $(0.40 ; 2.90)$ & 0.89 & 0.98 & $(0.36 ; 2.63)$ & 0.96 & 1.08 & $(0.40 ; 2.92)$ & 0.88 \\
Lag time above 95\% of reference & 1.29 & $(0.92 ; 1.79)$ & 0.14 & 1.25 & $(0.89 ; 1.75)$ & 0.20 & 1.17 & $(0.83 ; 1.65)$ & 0.36 \\
\hline ETP below 5\% of reference & 1.35 & $(0.94 ; 1.94)$ & 0.11 & 1.36 & $(0.94 ; 1.97)$ & 0.10 & 1.26 & $(0.87 ; 1.83)$ & 0.23 \\
ETP above 95\% of reference & 1.55 & $(1.11 ; 2.17)$ & 0.0099 & 1.53 & $(1.09 ; 2.15)$ & 0.015 & 1.50 & $(1.06 ; 2.13)$ & 0.023 \\
\hline Peak height below 5\% of reference & 1.07 & $(0.72 ; 1.59)$ & 0.76 & 1.09 & $(0.73 ; 1.63)$ & 0.66 & 1.02 & $(0.68 ; 1.52)$ & 0.92 \\
Peak height above 95\% of reference & 1.18 & $(0.78 ; 1.78)$ & 0.44 & 1.21 & $(0.80 ; 1.83)$ & 0.37 & 1.23 & $(0.81 ; 1.86)$ & 0.34 \\
\hline
\end{tabular}

Cox regression model performed in the overall study sample with mortality as outcome and markers of thrombin generation as predictors. Models were *adjusted for age, sex and vitamin $\mathrm{K}$ antagonist (VKA) use **adjusted for age, sex,VKA use and presence of cardiovascular risk factors (CVRF). ** adjusted for age, sex,VKA, use, presence of CVRF and cardiovascular disease.TG: thrombin generation; HR: hazard ratio; 95\% CI: 95\% confidence interval; ETP: endogenous thrombin potential.

However, these studies had rather small sample sizes and included a homogenous population of healthy volunteers. In the present analysis, age was positively associated with lag time in both males and females. In males, ETP and peak height increased with age, whereas in females the amount of TG showed a rather negative trend with less strong associations compared to those in males.

Other positive determinants of lag time observed in this study were obesity and dyslipidemia, which may partly be explained by increased levels of TFPI, a lipoproteinassociated coagulation inhibitor. It has been suggested that free TFPI is a major determinant of lag time. ${ }^{10}$ Elevated TFPI levels have been reported in individuals with impaired glucose tolerance and type 2 diabetes mellitus $^{20}$ and it has been suggested that these TFPI levels were elevated due to related obesity. ${ }^{21}$ Smid and colleagues found that a prolongation in lag time in patients with previous myocardial infarction may be due to release of TFPI. ' In addition to lag time, both ETP and peak height showed positive associations with obesity and dyslipidemia. Total body fat percentage and body mass index have been positively associated with lag time, ETP and peak height in females, independently of age, prior CVD, glucose metabolism and smoking status, though no associations were observed in males. ${ }^{22}$ The present study demonstrates strong relations of obesity with a longer lag time and higher ETP and peak height in both males and females, independently of potential confounders. The association of ETP and peak height with obesity may be attributed to a low-grade inflammation observed in obese individuals. ${ }^{23}$

The results on associations between therapy and TG parameters showed that use of vitamin $\mathrm{K}$ antagonists was positively associated with lag time and negatively associ- ated with ETP and peak height, as expected from previous studies. ${ }^{24-26}$ Aspirin showed no effect on TG (data not shown), in line with recent findings from the COMPASS trial, in which treatment with a combination of aspirin and rivaroxaban, a direct factor Xa inhibitor, showed a superior effect on prevention of the manifestation of atherothrombosis in atherosclerotic disease, as compared to treatment with aspirin alone. ${ }^{17}$

Furthermore, intake of oral contraceptives or hormone replacement therapy was associated with a shorter lag time and higher ETP and peak height, in line with previous reports..$^{10,27-29}$ The influence of estrogen-containing medication on the TG potential has been linked through increased levels of the coagulation factors II, VII, VIII, and $\mathrm{X}$ and fibrinogen, decreased levels of the natural anticoagulants, antithrombin and protein $S$, and acquired resistance to activated protein $\mathrm{C}^{27,28}$

Hitherto, only a limited number of studies have explored the association between TG and mortality. The PROSPER study, including only elderly individuals, showed positive associations of vascular mortality with lag time and peak height and total mortality with lag time. ${ }^{5}$ However, after adjustment for interleukin- 6 and Creactive protein levels, the associations were no longer significant, indicating that inflammation may be contributing to higher TG in these individuals. In another smaller study, higher ETP and peak height (at 5 pM TF), independently of age, sex and CVRF, were associated with increased risk of cardiovascular death in patients with acute coronary syndrome. ${ }^{30}$ In the present large, adult, population-based study, we demonstrate a positive association between lag time at 1 pM TF and total mortality, which remained significant after adjusting for traditional CVRF and CVD. Furthermore, this study highlights the 

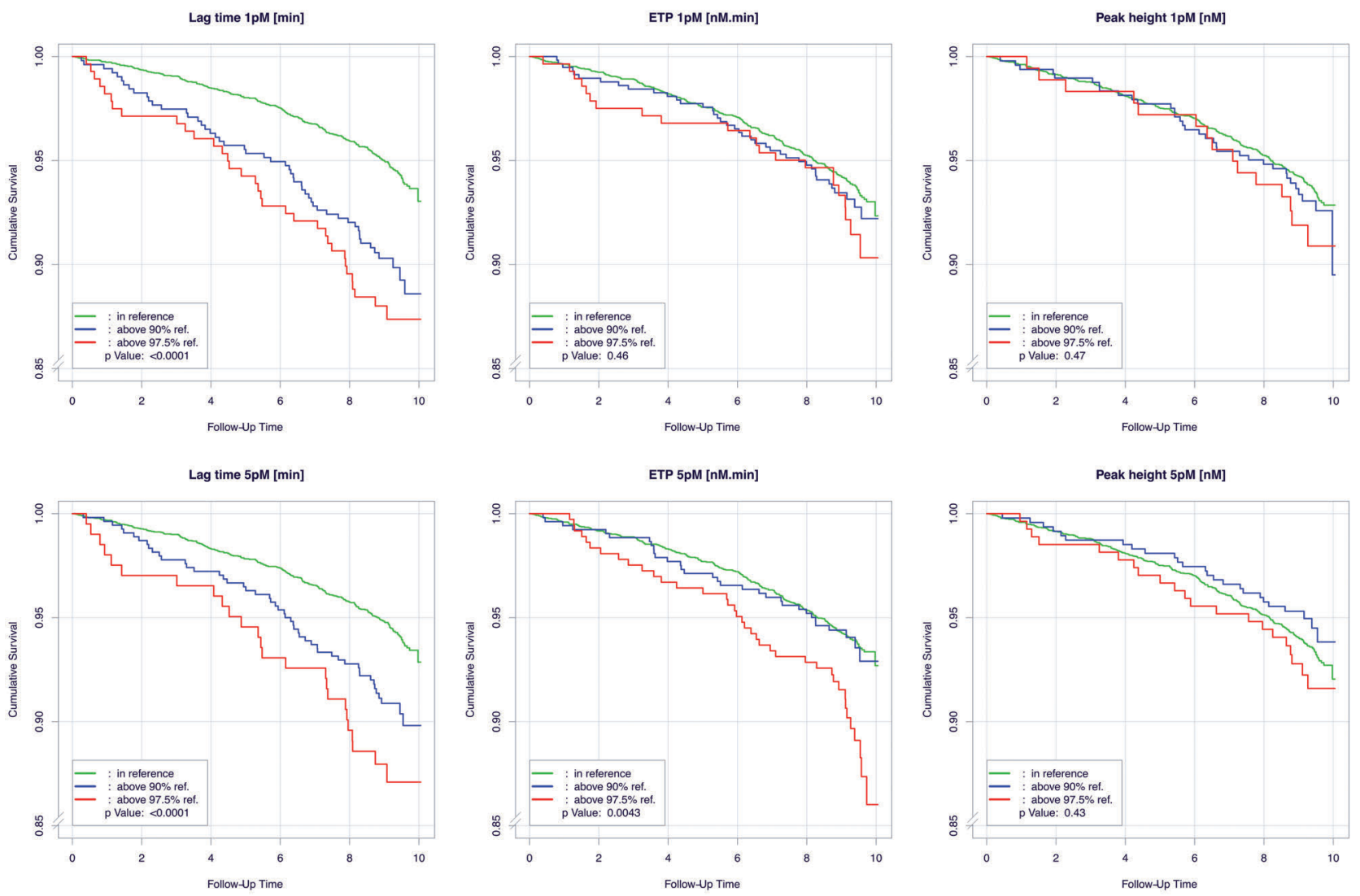

Figure 1. Survival over 10 years for markers of thrombin generation above and below reference limits. Kaplan-Meier survival curves of the overall study sample demonstrating the 10-year survival of individuals with the thrombin generation parameters lag time (left panels), endogenous thrombin potential (ETP) (middle panels), and peak height (right panels) within the range of the reference group (green line), individuals above the $90^{\text {th }}$ percentile of the reference group (blue line), and individuals above the $97.5^{\text {th }}$ percentile of the reference group (red line), at 1 (upper panels) and 5 pM (lower panels) tissue factor (TF). For the lag times at both 1 and $5 \mathrm{pM} \mathrm{TF}, P<0.001$ for the difference between the reference and the $90^{\text {th }}$ percentile, as well as for the reference and the $97.5^{\text {th }}$ percentile. For the ETP at 5 pM TF, $P=0.034$ for the difference between the reference and the $90^{\text {th }}$ percentile and $P=0.00097$ for the difference between the reference and the $97.5^{\text {th }}$ percentile.

relation between higher ETP (above the $95^{\text {th }}$ percentile of the reference group) at $5 \mathrm{pM}$ TF, as a global measure of both procoagulant and anticoagulant forces in the plasma, and increased risk of death, independently of CVRF and CVD. These findings indicate that both lag time and ETP are potential biomarkers for increased mortality risk, beyond the traditional CVRF. As discussed for the previous published PROSPER study, the association between a prolonged lag time and total mortality is not only a surprising and counterintuitive observation, but also one that is difficult to explain. With a risk of being too speculative, potential mechanisms might include consumption of initiators of coagulation before the system overshoots to start actual thrombosis. In other words, a constant (weak) prothrombotic trigger activates the coagulation system which is subsequently downregulated by the natural anticoagulants antithrombin and TFPI until, at a certain moment, the prothrombotic trigger increases and the system becomes overactivated and anticoagulants can no longer prevent thrombosis. In such a scenario, consumption of factor VII or XII, for example, could lead to a prolonged lag time in a sensitive in vitro assay. Another potential contributor to the prolonged lag time could be altered TFPI levels between subjects. However, assessing the TFPI levels in the presented cohort is part of another study and beyond the scope of the current study.
Limitations of our study are that we measured TG in platelet-poor plasma after one-step centrifugation of whole blood (10 min at 2,000 $\mathrm{xg}$ ) in contrast to recommendations (two-step centrifugation, $2000 \mathrm{x} \mathrm{g}$ for $5 \mathrm{~min}, 10,000 \mathrm{x} \mathrm{g}$ for 10 minutes). A previous small-scale analysis by Loeffen and colleagues ${ }^{16}$ showed that in order to eliminate residual platelets and microparticles, which may contribute to variability in TG results, double-centrifuged samples are preferable. We cannot, therefore, exclude that residual platelets and microparticles contributed to the observed associations between CVRF and TG parameters. Next, we had only cumulative mortality data available, so conclusions could not be made regarding associations between TG variables and specific causes of mortality. However, the standardized clinical investigation of the cardiovascular profile, standardized laboratory measurements of the large Gutenberg Health Study sample and availability of prospective mortality data are essential strenghts of our study, which delivers important evidence on the TG assay as a potential tool for improving risk stratification for CVD.

In conclusion, this is the first, large, population-based study demonstrating an important relation between TG parameters, such as the time to minimum thrombin formed or the amount of thrombin formed, and total mortality. Further research is required on the underlying mechanism as well as to explore the potential role of the 
parameters as independent biomarkers for increased mortality risk. The observed association of TG and traditional CVRF, particularly obesity, is an important finding in light of the growing "globesity" issue worldwide. ${ }^{31}$

\section{Acknowledgments}

We are indebted to all study participants and all co-workers of the Gutenberg Health Study, who were involved in the planning and conduct of this study.

\section{Funding}

The Gutenberg Health Study is funded through the govern- ment of Rhineland-Palatinate ("Stiftung RheinlandPfalz für Innovation", contract AZ 961-386261/733), the research programs "Wissen schafft Zukunft" and "Center for Translational Vascular Biology (CTVB)" of the Johannes GutenbergUniversity of Mainz, and its contract with Boehringer Ingelheim and PHILIPS Medical Systems, including unrestricted grants for the Gutenberg Health Study. This work was supported by the German Federal Ministry of Education and Research (BMBF 01E01003) and the Center for Translational Vascular Biology (CTVB) of the University Medical Center Mainz (to PSW). HtC is a Fellow of the Gutenberg Research Foundation.

\section{References}

1. Spronk HM, Govers-Riemslag JW, ten Cate $\mathrm{H}$. The blood coagulation system as a molecular machine. Bioessays. 2003;25(12): 1220-1228.

2. Dargaud $\mathrm{Y}$, Beguin S, Lienhart A, et al. Evaluation of thrombin generating capacity in plasma from patients with haemophilia A and B. Thromb Haemost. 2005;93(3):475480.

3. Besser M, Baglin C, Luddington R, van Hylckama Vlieg A, Baglin T. High rate of unprovoked recurrent venous thrombosis is associated with high thrombin-generating potential in a prospective cohort study. J Thromb Haemost. 2008;6(10):1720-1725.

4. Loeffen R, van Oerle R, Leers MP, et al . Factor XIa and thrombin generation are elevated in patients with acute coronary syndrome and predict recurrent cardiovascular events. PLoS One. 2016;11(7): e0158355.

5. Loeffen R, Winckers K, Ford I, et al. Associations between thrombin generation and the risk of cardiovascular disease in elderly patients: results from the PROSPER study. J Gerontol A Biol Sci Med Sci. 2015;70(8):982-988.

6. Lutsey PL, Folsom AR, Heckbert SR, Cushman M. Peak thrombin generation and subsequent venous thromboembolism: the Longitudinal Investigation of Thromboembolism Etiology (LITE) study. J Thromb Haemost. 2009;7(10):1639-1648.

7. Smid M, Dielis AW, Spronk HM, et al. Thrombin generation in the Glasgow Myocardial Infarction Study. PLoS One. 2013;8(6):e66977.

8. ten Cate-Hoek AJ, Dielis AW, Spronk HM, et al. Thrombin generation in patients after acute deep-vein thrombosis. Thromb Haemost. 2008;100(2):240-245.

9. Hemker HC, Giesen P, AlDieri R, et al. The calibrated automated thrombogram (CAT): a universal routine test for hyper- and hypocoagulability. Pathophysiol Haemost Thromb. 2002;32(5-6):249-253.

10. Dielis AW, Castoldi E, Spronk HM, et al. Coagulation factors and the protein $\mathrm{C}$ system as determinants of thrombin generation in a normal population. J Thromb Haemost. 2008;6(1):125-131.

11. Haidl H, Cimenti C, Leschnik B, Zach D,
Muntean W. Age-dependency of thrombin generation measured by means of calibrated automated thrombography (CAT). Thromb Haemost. 2006;95(5):772-775.

12. Chaireti R, Gustafsson KM, Bystrom B, Bremme K, Lindahl TL. Endogenous thrombin potential is higher during the luteal phase than during the follicular phase of a normal menstrual cycle. Hum Reprod. 2013;28(7):1846-1852.

13. Wild PS, Zeller T, Beutel $M$, et al. [The Gutenberg Health Study]. Bundesgesundheitsblatt Gesundheitsforschu ng Gesundheitsschutz. 2012;55(6-7):824-829.

14. Schnabel RB, Wilde S, Wild PS, Munzel T, Blankenberg S. Atrial fibrillation: its prevalence and risk factor profile in the German general population. Dtsch Arztebl Int. 2012;109(16):293-299.

15. Wild PS, Sinning CR, Roth A, et al. Distribution and categorization of left ventricular measurements in the general population: results from the population-based Gutenberg Heart Study. Circ Cardiovasc Imaging. 2010;3(5):604-613.

16. Loeffen R, Kleinegris MC, Loubele ST, et al. Preanalytic variables of thrombin generation: towards a standard procedure and validation of the method. J Thromb Haemost. 2012;10(12):2544-2554.

17. Eikelboom JW, Connolly SJ, Bosch J, et al. Rivaroxaban with or without aspirin in stable cardiovascular disease. N Engl J Med. 2017:377(14):1319-1330.

18. Lowe GD, Rumley A, Woodward M, et al. Epidemiology of coagulation factors, inhibitors and activation markers: the Third Glasgow MONICA Survey. I. Illustrative reference ranges by age, sex and hormone use. Br J Haematol. 1997;97(4):775-784.

19. Spronk HM, Dielis AW, De Smedt E, et al. Assessment of thrombin generation II: validation of the Calibrated Automated Thrombogram in platelet-poor plasma in a clinical laboratory. Thromb Haemost. 2008;100(2):362-364.

20. Leurs PB, Stolk RP, Hamulyak K, Van Oerle $\mathrm{R}$, Grobbee DE, Wolffenbuttel BH. Tissue factor pathway inhibitor and other endothelium-dependent hemostatic factors in elderly individuals with normal or impaired glucose tolerance and type 2 diabetes. Diabetes Care. 2002;25(8):13401345 .

21. Vambergue A, Rugeri L, Gaveriaux V, et al.
Factor VII, tissue factor pathway inhibitor and monocyte tissue factor in diabetes mellitus: influence of type of diabetes, obesity index, and age. Thromb Res. 2001;101(5): 367-375.

22. Beijers HJ, Ferreira I, Spronk HM, et al. Body composition as determinant of thrombin generation in plasma: the Hoorn study. Arterioscler Thromb Vasc Biol. 2010;30(12): 2639-2647.

23. Pruller F, Raggam RB, Posch V, et al. Trunk weighted obesity, cholesterol levels and low grade inflammation are main determinants for enhanced thrombin generation. Atherosclerosis. 2012;220(1):215-218.

24. Brodin E, Selieflot I, Arnesen H, Hurlen M, Appelbom H, Hansen JB. Endogenous thrombin potential (ETP) in plasma from patients with AMI during antithrombotic treatment. Thromb Res. 2009;123(4):573579.

25. Wielders S, Mukheriee M, Michiels I, et al The routine determination of the endogenous thrombin potential, first results in different forms of hyper- and hypocoagulability. Thromb Haemost. 1997;77(4):629-636.

26. Gatt A, van Veen JJ, Bowyer A, et al. Wide variation in thrombin generation in patients with atrial fibrillation and therapeutic International Normalized Ratio is not due to inflammation. Br J Haematol. 2008;142(6): 946-952.

27. Rotteveel RC, Roozendaal KJ, Eijsman L, Hemker HC. The influence of oral contraceptives on the time-integral of thrombin generation (thrombin potential). Thromb Haemost. 1993;70(6):959-962.

28. Tchaikovski SN, van Vliet HA, Thomassen $\mathrm{MC}$, et al. Effect of oral contraceptives on thrombin generation measured via calibrated automated thrombography. Thromb Haemost. 2007;98(6):1350-1356.

29. Marchi R, Marcos L, Paradisi I. Comparison by sex between thrombin generation and fibrin network characteristics in a healthy population. Clin Chim Acta. 2015;441:8689

30. Attanasio M, Marcucci R, Gori AM, et al. Residual thrombin potential predicts cardiovascular death in acute coronary syndrome patients undergoing percutaneous coronary intervention. Thromb Res. 2016;147:52-57.

31. James PT, Leach R, Kalamara E, Shayeghi M The worldwide obesity epidemic. Obes Res. 2001:9(Suppl 4):228S-233S 